\title{
Enteric disease outbreak reporting, New South Wales, Australia, 2000 to 2005
}

\section{Michelle Cretikos ${ }^{\mathrm{A}, \mathrm{C}}$, Barbara Telfer $^{\mathrm{B}}$ and Jeremy McAnulty ${ }^{\mathrm{B}}$}

ANSW Public Health Officer Training Program, NSW Department of Health

${ }^{\mathrm{B}}$ Communicable Diseases Branch, NSW Department of Health

${ }^{\mathrm{C} C o r r e s p o n d i n g}$ author: Email:mcret@doh.health.nsw.gov.au.

\begin{abstract}
Objective: To review enteric disease outbreaks reported to the NSW Department of Health. Methods: Data from existing electronic enteric disease outbreak summary databases were used to describe the number and type of outbreaks reported, burden of illness and cause of the outbreaks. Results: Between 2000 and 2005, 998 enteric disease outbreaks were reported (148 foodborne and 850 non-foodborne), affecting 24260 people and associated with 771 hospitalisations and 21 deaths. Salmonella was confirmed in 28 per cent of foodborne outbreaks, and norovirus in 18 per cent of non-foodborne outbreaks.
\end{abstract}

Conclusions: Enteric disease outbreaks cause a substantial burden of disease in NSW.

While many infectious agents can cause outbreaks, most of the outbreaks reported in NSW involve enteric diseases, which are caused by the ingestion of infectious agents or toxins, and result in nausea, vomiting, diarrhoea, abdominal cramps and fever, or other symptoms. ${ }^{1,2}$

Enteric diseases place a substantial burden on the community. It has been estimated that in Australia there are approximately 17.2 million cases of gastroenteritis each year. ${ }^{3}$ A national survey of gastroenteritis in Australia during 2001-2002 found that one-third of working adults miss at least one day of work when they have gastroenteritis, and one-third of cases result in a caregiver missing work. ${ }^{3}$

Of these gastroenteritis cases, 5.4 million are estimated to be cases of foodborne-associated gastroenteritis, equivalent to 0.3 episodes of foodborne gastroenteritis per person per year, and resulting in approximately 18000 hospitalisations and 120 deaths in Australia annually. ${ }^{4}$ Many out- breaks of gastroenteritis occur in institutions, such as schools, childcare centres and residential care facilities. Institutional outbreaks of gastroenteritis are usually caused by highly infectious viruses such as norovirus and rotavirus, and spread predominantly through person-toperson contact. ${ }^{1,5}$

Under the Public Health Act (NSW) 1991, 12 enteric conditions are currently notifiable in NSW and must be reported to NSW Health by doctors, laboratories and hospitals, including: botulism, cholera, cryptosporidiosis, giardiasis, haemolytic uraemic syndrome (HUS), shigatoxigenic Escherichia coli (STEC) infection, hepatitis A, hepatitis E, listeriosis, salmonellosis, shigellosis and typhoid. Some of these diseases are primarily foodborne in origin, such as salmonellosis, typhoid and listeriosis.

In addition to individual disease notifications, outbreaks of suspected foodborne disease in two or more people related in time or place, and outbreaks of gastroenteritis among people of any age in an institution (eg an educational, residential, childcare or healthcare institution) are also notifiable by doctors and institutions. Where public health units identify clusters of disease or outbreaks, they will launch an outbreak investigation. Summary reports of enteric disease outbreaks are provided by public health units and entered into the relevant enteric disease outbreak databases held at the NSW Department of Health.

We analysed enteric disease outbreaks reported to the NSW Department of Health for the five-year period July 2000-June 2005. This analysis aimed to describe the number, epidemiology and cause of the enteric disease outbreaks reported during this period.

\section{Methods}

The two existing NSW Health enteric disease outbreak databases - the OzFoodNet Outbreak Summary Database and the Gastroenteritis in Institutions Database - were used. For the purposes of the present study, the data contained within the two enteric disease outbreak summary databases was separated into two categories:

- foodborne and suspected foodborne outbreaks, and

- non-foodborne outbreaks of enteric disease.

If the setting of the outbreak was an institution (ie aged care facility, hospital, child care centre, school, correctional facility or other institutional setting) and the method of transmission was unknown, the outbreak was assumed 
Table 1. Number and type of enteric disease outbreaks, New South Wales, 2000-2005

\begin{tabular}{lcccc}
\hline Financial year & $\begin{array}{c}\text { Foodborne or suspected foodborne } \\
\text { Institutional } \\
n\end{array}$ & $\begin{array}{c}\text { Non-institutional } \\
\boldsymbol{n}\end{array}$ & $\begin{array}{c}\text { Non-foodborne } \\
\text { Institutional } \\
\boldsymbol{n}\end{array}$ & $\begin{array}{c}\text { Non-institutional } \\
\boldsymbol{n}\end{array}$ \\
\hline $2000-01$ & 0 & 25 & 43 & 7 \\
$2001-02$ & 1 & 36 & 51 & 11 \\
$2002-03$ & 2 & 28 & 121 & 21 \\
$2003-04$ & 11 & 26 & 390 & 9 \\
$2004-05$ & 0 & 19 & 181 & 64 \\
Total & 14 & 786 & \\
\hline
\end{tabular}

to be a non-foodborne outbreak unless Salmonella, which is commonly transmitted by the foodborne route, was identified. Only two Salmonella outbreaks in institutions were attributed to a foodborne route as a result of this decision. For the purposes of analysis, all foodborne and suspected foodborne outbreaks (including those occurring in an institutional setting) were considered to be foodborne outbreaks. All other outbreaks were considered to be nonfoodborne outbreaks.

The number and type of enteric disease outbreaks, the reported number of people affected, the reported number of hospitalisations, the reported number of deaths and the annual rate of enteric disease outbreaks reported by NSW public health units for each 100000 population were estimated. Mode of transmission, extent of spread and cause were also examined.

An enteric agent (organism or toxin) was considered to have caused the outbreak if it was identified through microbiological testing of a clinical specimen and/or the implicated food vehicle and the epidemiologic features were consistent with the features of disease caused by the agent.

Tests of proportions were performed using the chi-square test. Tests of duration of illness were performed using the Kruskal-Wallis test.

\section{Results}

Between 1 July 2000 and 30 June 2005, 998 enteric disease outbreaks were reported in NSW (Table 1). The number of foodborne outbreaks reported each year was reasonably stable, with an average of 30 ( 15 per cent of all outbreaks). The number of reported non-foodborne outbreaks increased substantially between 2000 and 2005, with an epidemic year of viral gastroenteritis in 2004 (Figure 1). The majority of non-foodborne outbreaks occurred in institutions.

These outbreaks affected 24260 people. At least 2072 people ( 8.5 per cent) reported seeing a doctor as a result of

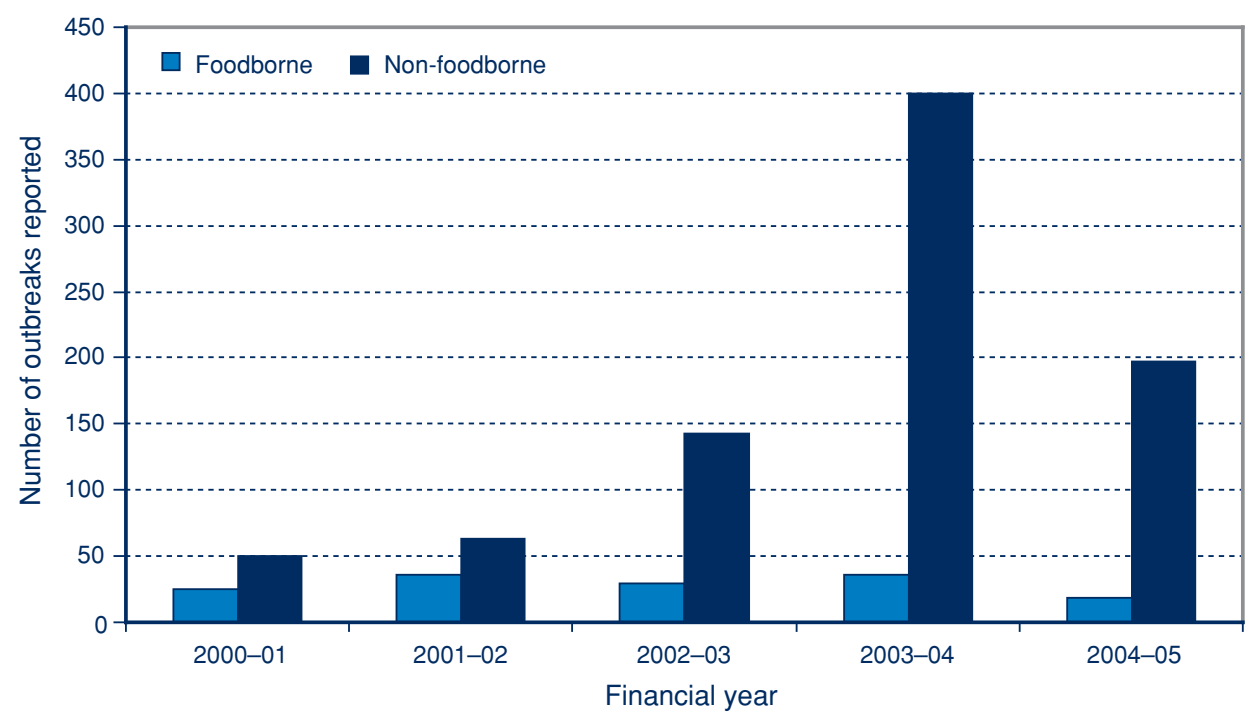

Figure 1. Total number of enteric disease outbreaks reported by financial year in New South Wales, 2000-2005. Source: Gastroenteritis in Institutions Database; OzFoodNet Outbreak Summary Database. 
Table 2. Number of people affected, hospitalised and dying in association with enteric disease outbreaks, New South Wales, 2000-2005

af those affected

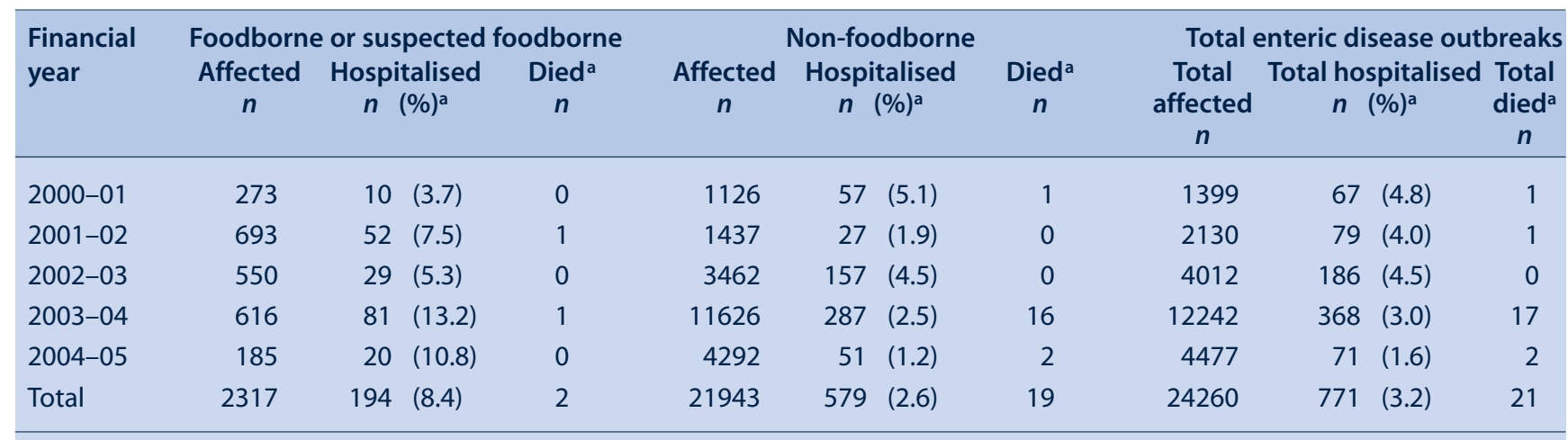

Source: Gastroenteritis in Institutions Database; OzFoodNet Outbreak Summary Database.

their illness, 771 people (3.2 per cent) required hospitalisation and 21 people $(<0.1$ per cent) died (Table 2$)$.

The median duration of reported illness in affected people was 1.5 days (inter-quartile range (IQR) 1-2 days). The median duration was significantly longer for foodborne illness (2 days, IQR 1-4 days) than for non-foodborne illness ( 1.3 days, IQR $1-2$ days, $p<0.001)$. In 17 of the outbreaks, several people were still reported to be ill at the time the summary report was provided, which indicates the true duration of illness would be longer than that reported.

Among the foodborne outbreaks, 14 of the 198 non- institutional outbreaks were quite widespread, with five multi-state outbreaks and nine outbreaks involving multiple Area Health Service regions within NSW. Nine of these 14 multi-region outbreaks were reported to be caused by various Salmonella species. In the remainder, the cause was unknown. The most commonly reported mode of transmission of non-foodborne outbreaks was person-to-person (320/850 person-to-person: 37.6 per cent; 452/850 suspected person-to-person: 53.2 per cent).

The total rates of reporting of enteric disease outbreaks by public health unit were highly variable, ranging from 0.5 to 7.4 per 100000 population (Figure 2).

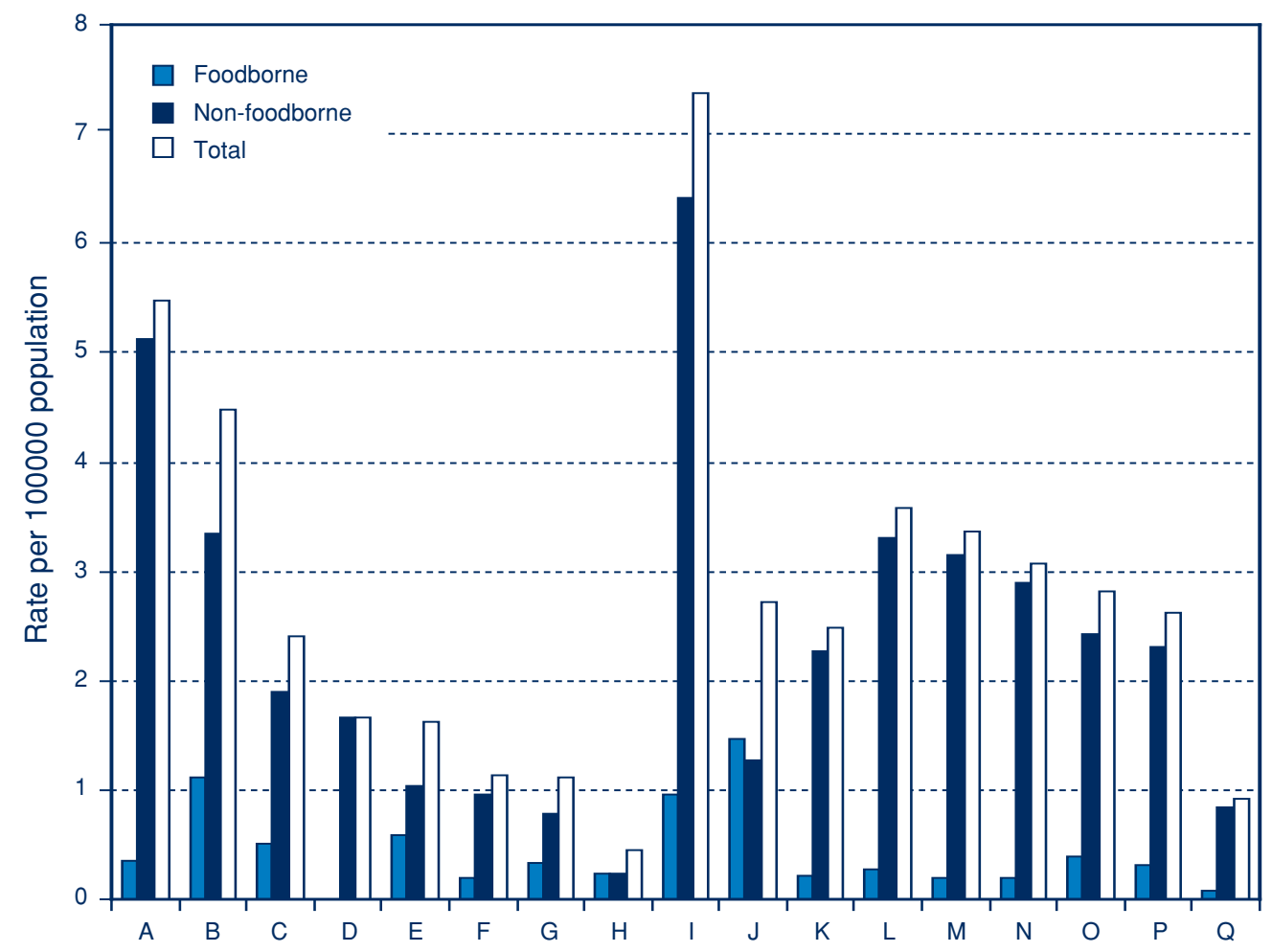

Figure 2. Rate of reporting of enteric disease outbreaks in New South Wales by public health unit, 2000-2005. Source: Gastroenteritis in Institutions Database; OzFoodNet Outbreak Summary Database. 
Table 3. The reported cause of enteric disease outbreaks, New South Wales, 2000-2005

\begin{tabular}{lccc}
\hline Reported organism/toxin & $\begin{array}{c}\text { Foodborne } \\
n\end{array}$ & $\begin{array}{c}\text { Non-foodborne } \\
n\end{array}$ & $\begin{array}{c}\text { Total } \\
n\end{array}$ \\
\hline Ciguatoxin & 1 & 0 & 1 \\
Hepatitis A & 1 & 0 & 1 \\
Vibrio parahaemolyticus & 1 & 0 & 1 \\
Blastocystis hominis & 0 & 1 & 1 \\
Cryptosporidium & 0 & 1 & 1 \\
Dientamoeba fragilis & 0 & 1 & 1 \\
Clostridium difficile & 0 & 2 & 2 \\
Giardia & 0 & 2 & 2 \\
Clostridium perfringens & 3 & 0 & 3 \\
Scombrotoxin & 3 & 0 & 3 \\
Toxin of unspecified type & 4 & 0 & 4 \\
Campylobacter jejuni & 2 & 2 & 4 \\
Both norovirus and rotavirus & 0 & 4 & 25 \\
Rotavirus & 1 & 24 & 44 \\
Salmonella species & 42 & 2 & 152 \\
Norovirus & 2 & 150 & 749 \\
Unknown & 88 & 661 & 998 \\
Total & 148 & 850 & \\
\hline Source: Gastroenteritis in Institutions Database; OzFoodNet Outbreak Summary Database. & &
\end{tabular}

At least one stool specimen was reported to have been collected in a significantly smaller proportion of foodborne (54/148: 36 per cent) than non-foodborne outbreaks (391/850: 46 per cent, $p=0.02)$. The causative agent for the outbreak was determined in a significantly larger proportion of the foodborne (60/148; 41 per cent) than non-foodborne outbreaks (189/850: 22 per cent, $p<$ 0.001). By far the most common cause of the outbreaks was salmonellosis for foodborne outbreaks, and norovirus and rotavirus for non-foodborne outbreaks (Table 3).

\section{Discussion}

Enteric disease outbreaks create a substantial burden of illness in NSW, but these outbreaks rarely result in severe illness or death. The majority of the enteric disease outbreaks reported during 2000-2005 were non-foodborne institutional outbreaks of viral gastroenteritis, particularly during the epidemic year of 2004. These outbreaks affected many thousands of people and were associated with a small number of deaths. Rapid control of viral institutional outbreaks should be a priority in order to prevent such large institutional outbreaks in the future.

There are several limitations to the present study. First, given that there are approximately 17.2 million cases of gastroenteritis in Australia annually, it is likely that many enteric disease outbreaks that occur in NSW are not detected or reported. ${ }^{3}$ This indicates that these results are likely to be an underestimate of the true burden of disease from enteric disease outbreaks in NSW. Second, the rate of reporting of enteric disease outbreaks is also inconsistent in public health units, which suggests that the enteric disease outbreaks reported do not represent the true distribution of disease in NSW. Finally, the mode of transmission for the majority of the outbreaks was unconfirmed, making the determination of a transmission mode uncertain.

It is important to note that the public health unit with the highest rate of reporting of enteric disease outbreaks (PHU I) is the only unit in NSW that has staff funded exclusively to perform enteric disease surveillance and control activities. This may account for the high rates of reporting of enteric disease outbreaks from this area. Other reasons for the variation in outbreak reporting are unclear, but may include: variations in public health unit capacity due to other competing public health activities; the number of staff trained to investigate enteric diseases; the number of institutions, such as aged care facilities, in each area; the level of notification from institutions and the community; and other local characteristics, including exposure to farm animals, water quality and other environmental factors.

\section{Conclusion}

Given the burden of illness caused by enteric disease outbreaks, the identification, investigation, control and prevention of enteric disease outbreaks in NSW are important public health activities. 


\section{Acknowledgements}

Thanks to Jennie Musto for reviewing earlier versions of this document and providing valuable feedback. Thanks also to all the public health unit staff who provided outbreak summary reports during the period of the evaluation.

\section{References}

1. NSW Department of Health Year in Review: Communicable Disease Surveillance, 2004. N S W Public Health Bull 2005; 16: 77-87. doi:10.1071/NB05019

2. OzFoodNet Working Group Reported foodborne illness and gastroenteritis in Australia: Annual report of the OzFoodNet network, 2004. Commun Dis Intell 2005; 29: 165-85.
3. Hall G. OzFoodNet Working Group. Results of the national gastroenteritis survey 2001-2: working paper series no. 50. Canberra: National Centre for Epidemiology and Population Health, 2004.

4. Hall G, Kirk MD. OzFoodNet Working Group. Foodborne illness in Australia: Annual incidence circa 2000. Canberra: Australian Government Department of Health and Ageing, 2005.

5. Department of Health SA. Guidelines for the management of infectious gastroenteritis in aged care facilities in South Australia. Adelaide: Communicable Disease Control Branch, 2005.

\section{NSW Public Health Bulletin Subscription Form and Electronic Early Alert Service}

To subscribe to the hard copy of the NSW Public Health Bulletin or to change your subscription details please complete this form and return it by email (phbulletin@doh.health.nsw.gov.au) or fax (61 293919232 ).

The Bulletin can be accessed electronically from www.publish.csiro.au/journals/phb. Subscribe to the Early Alert service to be notified as soon as it appears online (http://publish.csiro.au/nid/226.htm?nid=25\&aid=685).

\section{Subscription information}

I wish to receive the NSW Public Health Bulletin:

My details are as follows

Name:

Organisation:

Mailing Address:

State: Postcode: Country:

Telephone: Facsimile:

Email:

\section{Change of Address}

I wish to change my mailing details, as follows:

Name:

From: [Insert old address]

To: [Insert new address] 